Steven SMITH

North Carolina State University

Raleigh, North Carolina

slsmit17@ncsu.edu

\title{
BRAIDED ENVIRONMENTS: A CALL TO EXPLORE RHETORIC AND MATERIALITY THROUGH AUGMENTATIVE TECHNOLOGIES
}

Recommended Citation: Smith, Steven. "Braided Environments. A Call to Explore Rhetoric and Materiality through Augmentative Technologies." Metacritic Journal for Comparative Studies and Theory 4.2 (2018): https://doi.org/10.24193/mjcst.2018.6.01

\begin{abstract}
This research proposes that augmented reality technologies have the capabilities of intertwining natural spaces, material spaces, and networked (or immaterial) spaces together through the author's original idea of the between, which can in turn be used to further explore rhetorical discourse and im/materiality and bolster emerging discourse within the digital humanities. By using a variety of contemporary research on diverse topics within augmented reality and the humanities, such as the inside/outside model and the regenerative/transformative concept (each proposed by Victoria Gallagher in her works on visual rhetoric), as well as an examination of current augmented reality applications (Wikitude and Pokémon Go), the author argues that emerging applications in AR can offer scholars a variety of possibilities to explore new media and the humanities, as well as more interdisciplinary fields such as public spaces, natural spaces, digital spaces, rhetoric, science, technology, and critical cultural theory.
\end{abstract}

Keywords: Material rhetoric, augmented reality, Pokémon Go, Wikitude, virtual community, game studies, posthumanism

In 2012, North Carolina State University Communication scholars Victoria Gallagher, Kenneth Zagacki, and Kelly Norris in their chapter Material and Urban Communication: The rhetoric of Communicative Spaces (part of a much larger 
collection of communication and materiality research found within the book Communication Matters) explore how "the material and symbolic features of [a] park create a space that is both inside and outside ${ }^{1}$ of the urban experience and of the city's history, which demonstrates the possibilities of the human-nature, human-human and human-urban interface" and "how the park situates visitors as engaging in modes of performance, related to interacting and feeling" (Gallagher, "Materiality" 108). While the goal of their research was to "examine the spaces within cities that enable citizens to engage communicatively" and to analyse "the extent to which parks may function as performative spaces," the goal of this research paper is to extend their idea of the inside/outside to include networked, or virtual, spaces, and do so in light of augmented reality technologies (Gallagher, "Materiality" 109). First, however, I wish to continue with a brief analysis of Gallagher et al.'s work.

The chosen medium for the work of Gallagher et al. was Chicago's Millennium Park, which offers a "reflection of nearly two centuries of Chicago history, politics and culture" via the park's several monuments and sculptures (Gilfoyle xiii). What the authors found in their rhetorical analysis of Millennium Park was that various architectural elements, or sculptures, of the park could be explored through their proposed inside/outside model. Cloud Gate, more commonly referred to as simply "The Bean," for example, offers to its viewer a means to view what is both inside of and outside of the park because of its reflective surface. The Crown Fountain, two 50 feet tall fountains found on Michigan Avenue, allows visitors to go inside, where a camera displays their faces on the outside of the tower and spouts water out of their "video" portraits' mouths; this literally puts visitors "inside" the fountain's tower, which allows them to interact with those "outside" that are in the reflecting pools of the video sculpture. The Jay Pritzker Music Pavilion and Great Lawn operate as both "an outdoor (emphasis added) music stage and an indoor reception area" intertwining man-made and natural elements within the Millennium Park (Gallagher, "Materiality" 115).

What Gallagher, Zagacki, and Martin found was that

\footnotetext{
${ }^{1}$ As defined by Zagacki and Gallagher: "The experience of moving 1) between constructed spaces, such as a museum space or urban landscape, to less constructed, more organic spaces, such as the outdoor park or the rural landscape; and 2) between natural history and human history." (Galagher "Rhetoric" 173)
} 
the park's elements, taken together, are characterized by their highly interactive nature: visitors perform certain types of relationship between themselves and the cityscape around them as well as between themselves and other citizens. In so doing, they experience their city from a much richer and varied set of sensibilities, opening them up to interactions and new perspectives (Gallagher, "Materiality" 116).

Their examination of various sculptures and artwork found at the Millennium Park may break away in regard to traditional rhetorical analyses, such as speeches, but ultimately Gallagher, Zagacki, and Martin, through their exploration of urban settings, helped push the envelope of rhetorical discourse further to include material objects and blend them with the natural environment.

A second article by Victoria Gallagher and Kenneth Zagacki, titled "Rhetoric and Materiality in the Museum Park at the North Carolina Museum of Art," further built upon their previous work in material rhetoric, this time by examining a park local to their home institution in Raleigh, North Carolina. While focusing again on the inside/outside components of material rhetoric, they also introduce the regenerative/transformative ${ }^{2}$ concept of conceptualizing rhetoric. Their goal in this research was similar to that of the previous project, in which they "provide a critical analysis of how the inside/outside and regenerative/transformative enactments of the Museum Park engage visitors rhetorically, thereby creating spaces of attention in which they can consider and experience the human/nature interface in new ways" (Gallagher, "Rhetoric" 173). As a result of their analysis, they conclude that "discussing the extent to which the material rhetoric of museum parks may lead to the development of environmental consciousness and community identity, as well as the tensions associated with this process" (Gallagher, "Rhetoric" 173). In other words, Gallagher and Zagacki propose that spaces which engage hearing, touch, and sight offer the possibility of raising environmental awareness and community identity.

Gallagher and Zagacki focus on several sculptures found throughout Museum Park, all while applying the inside/outside concept. Their analysis of the sculpture Gyre, for example, examines "the experience of being inside and outside of nature

2 Defined by Gallagher and Zagacki as moving "1) from natural states to human-constructed states and back again to nature, and 2) from one state of understanding to another". 
simultaneously and of finding a balanced existence in this state," as the sculptures themselves are metal, upright "rings" that protrude through the surface of the earth (Galllagher, "Rhetoric" 177). This artwork offers viewers the capability to "mingle" and "intertwine" with the sculpture, as opposed to simply standing in front of it, allowing for an expanded experience beyond traditional monuments while reminding them of the power nature wields by altering the surface of the metallic rings of Gyre. To See Jennie Smile, another sculpture found at Museum Park, operates in an analogous way as Gyre, acting as a "mountain of paper [that] reveals for visitors the possibility that even as the materials we take from nature remain impermanent, they serve our purposes for periods of time" (Gallagher, "Rhetoric" 177-8). Mimicking the stump of a tree by penetrating the surface of the earth, To See Jennie Smile looks similar to a stack of newspapers, reminding viewers of the taxation they put on nature through their consumption of trees and paper.

Gallagher and Zagacki offer analyses of three other artifacts in their article, including Crossroads/Trickster, Cloud Chamber, and Picture This, each of which highlight the concepts of inside/outside and regenerative/transformative movements by alternating between human history and natural history and encompassing movement from a natural state, to a human-constructed states, and back again to nature. In each capacity, the sculptures demonstrate how the "Museum Park's main enactments contribute to forming spaces of attention" (Gallagher, "Rhetoric" 184). This allows and encourages individuals "to consider and reconsider their relationship with nature and, also, with each other," as well as enticing visitors "to become more actively selfconscious of the possibilities and pitfalls of this relationship" (Gallagher, "Rhetoric" 186-188). By exploring the interaction of sculptures that exhibit traits of the inside/outside concept, we can learn more about, and build upon, our relationship with nature.

Once again, Gallagher and Zagacki expanded upon the traditional model of rhetorical discourse in order to include material sculptures that penetrate the relationship between man and nature. By examining structures and public parks, which can create "spaces of attention," the authors argue we can learn more about our relationship with nature in hopes of becoming more aware of our impact on natural environments. 
The impact of Gallagher and Zagacki's concept of the inside/outside has been far reaching, and has helped to expand the possibility of what material rhetoric discourse may include. For example, in an article titled "Museums as Material: Experiential Landscapes and the Canadian Museum for Human Rights," Jennifer Clary-Lemon utilizes the inside/outside model to examine a sculpture within the CMHR - Trace. Trace is a "large ceramic blanket in the shape of a cloth draped on a hook" and sculpted out of Winnipeg-dug clay "in homage and reference to the excavation of the earth during the CMHR's construction, as well as to the archaeological material that was removed from the building site" (Clary-Lemon para. 24). Similar to sculptures analysed by Gallagher, Zagacki, and Martin, Trace operates inside/outside by intertwining, and allowing viewers to move between, natural elements (in this case, the Winnipeg clay) and a public space (CMHR). Dr. Vanessa Kraemer Sohan, an Assistant Professor of Writing \& Rhetoric at Florida International University in Miami, utilizes the inside/outside in her course titled "Material Rhetorics and Writing", in which she requires students to "situate the material rhetoric under consideration within conversations inside/outside of Rhetoric and Composition on materiality and culture" (Clary-Lemon para. 3).

As expansive as the inside/outside model has become in regard to material rhetoric discourse, given the current state of the advancement of augmented reality technologies, I believe there is room to build upon, or redefine, the inside/outside. Thus, throughout the remainder of this research, I will propose that implementing the use of augmentative applications has the capabilities of intertwining natural spaces, material spaces, and networked (or immaterial) spaces together, which can be used to further explore rhetorical discourse and im/materiality3. This will include exploring two augmented reality applications (Wikitude SDK and the augmented reality mobile application Pokémon Go), their usage, their relationship with natural and material spaces, how they transcend beyond the former spaces and into immateriality, and ultimately how they are interacted with through cyberspace. This will offer a new means to explore material rhetoric discourse - through the inclusion of networked environments.

3 By this I mean the exploration of how we interact with others in both material and immaterial settings. 


\section{On Digital Enhancements, Public Spaces, and Natural Environments}

The history and development of augmented reality (AR) is inextricably rooted with computer evolution. Since its introduction by Ivan Sutherland in 1968, augmented reality technologies have made only small strides; arguably the most successful implementation of AR technology for several years was the virtual "first down marker" during NFL broadcasts (that is, the yellow line that indicates a team's required yards to obtain a first down). Other strides made in the field include overlay tools that complemented video cameras or projectors being used to impact materiality with "virtual" images. It was not until relatively recently that the technology available could handle the demands augmented reality required.

The announcement of Google Glass in 2014 exhibited to the world the possibilities of augmented reality by superimposing materiality with superficiality on an accessible level, yet the project was ultimately halted for several reasons (safety concerns and cost over-run being the two most common explanations). While Google Glass is arguably responsible for pushing AR technology to the consumerism forefront, the mantle has since then been taken up by smart phones and easily-transportable devices, such as headsets. Augmented reality applications found and purchased through smart devices are designed to enhance real-world experiences across a variety of disciplines and job fields, while devices such as headsets are made to further capitalize on that enhancement.

The recent emergence of augmented reality has been an arising focal point for media and critical cultural scholars interested in posthumanism, visual rhetoric, virtual reality, new media, and digital design. In their 2010 book "Exploring Digital Design: Multi-Disciplinary Design Practices," authors Ina Wagner, Tone Bratteteig, and Dagny Stuedahl discuss the impact that augmented reality technologies could have on digital design in the humanities by superimposing virtual images on material surfaces. For example, how could the inclusion of augmented reality, wireless internet, GPS, motion tracking, and multimodal databases impact design practices, and how could this affect users seeking to learn from those technologies? Although the state of technology six years ago was significantly different than that of today, Wagner, Bratteteig, and Stuedahl believe that "the computer is a communicational device, a vehicle for 
mediation of multimodal textuality, meanings and messages" and should be utilized for construction and inspiration in digital textuality (Wagner et al 188).

Continuing the conversation of simulation and building off Wagner, Bratteteig, and Stuedahl, Gunnar Liestøl in his article "Augmented Reality and Digital Genre Design - Situated Simulations on the iPhone" explores the intersection between "rhetoric, genre, and practical digital design within the domain of augmented reality" through situated simulations and how they can aid the learning process (Liestøl 29). Defined as the "combination or juxtaposition of two different perspectives (real and virtual)", situated simulations work by creating a "double perspective, which makes it possible to present on the site topics or subject matters which are otherwise absent or invisible" (Liestøl 30). After exhibiting and analysing three different examples of situated simulation, the author believes that these types of augmented reality can "expand our understanding of both time and space", like how "change[s] over time and how it (time) may be influenced by human actions (Liestøl 33).

Wagner, Bratteteig, Stuedahl, and Liestøl's examination of augmentation and digital discourse theorize the capabilities that superimposing images on reality has by citing their own examples in digital design and how it could enhance learning. In "Exploring Digital Design", the authors use two examples of augmented reality on historical sites - one a gravemound of the Oseberg Viking Ship that has been excavated and put into a museum, the other the battle site of the Roman Civil War's Battle of Pharsalus - and how these technologies could allow viewers to see artifacts as they were once preserved in nature or view a simulation of the battle from various positions on the actual battlefield. Likewise, Liestøl's article offers examples of how augmentation can enhance learning. In one scenario, viewers examine Mission Dolores, San Francisco's oldest building, through augmentative techniques which allow the user to "navigate around and inside the now absent parts of the original construction, accessing audio and visual information about the historic landmark" (Liestøl 31). Another example from Liestøl shows the changing landscape of San Francisco during and after the earthquake and fire of 1906.

The above examples offer just a few possibilities of the impact that augmented reality could have on digital discourse. Each seeks to enhance the learning experience by offering viewers innovative ways to examine historical sites that have been altered 
through time by both man and nature, ultimately allowing them to see a kind of convergence between past and present; this helps to reinvent certain aspects of the humanities (visual rhetoric being just one example) and allow fields to go "beyond the book" to see and experience sites in new, informative ways.

Other than having been enhanced through augmented reality applications, the above examples have another thing in common: each offer the possibility of being analysed using the inside/outside concept introduced by Gallagher, Zagacki, and Martin, yet the opportunity to do so with the inclusion of augmented reality has been missed by rhetorical scholars interested in material discourse. In each scenario, the superimposed image occurs in tandem with a historic monument or landmark that is grounded in material rhetoric (the gravemound, the battle site, Mission Dolores, or the structural changes in San Francisco throughout 1906), and each can be examined through the inside/outside model. The inclusion of augmentation to that site, however, offers more capabilities of discursive analysis by adding an entirely new realm of exploration to which we can expand upon the inside/outside: between. The "between" in relation to the inside/outside would therefore be the internet "networks" that go "between" urban environments and organic spaces that ultimately flow into some online environment (like a virtual community or where online engagement takes place). This expands upon the possibilities that one could explore when considering landmarks by examining how they are interacted with through cyberspace.

Take, for example, Gyre, since it has already been examined by Gallagher and Zagacki through the application of inside/outside. Gyre exists both inside and outside of nature simultaneously by allowing viewers to move between a constructed space (the sculpture itself, inside) and back into nature (the park, outside). With the correct augmented reality application, Gyre's experience could be transcended beyond both natural and public spaces and into the digital realm where it could become the subject of an interactive learning experience, or simply be played with (as will be shown later), in a way not possible without simulation technologies. This is just what the creators of the mobile application "Wikitude" had in mind when developing their augmented reality services. As a mobile application, Wikitude's function is simple: to present the viewer with various points of interest (POI) in a given area that helps them to explore their surroundings more effectively. When the application is opened on a mobile device, it 
locates the user via GPS and then pinpoints attractions (up to 1.5 miles) that the user might be interested in on their mobile device's screen, as well as on a small compass in the upper right-hand corner of the application which indicates the direction each attraction is in. On top of that, Wikitude adds the option to route the person there (using Google Maps or something similar) and to access the Wikipedia webpage of the attraction so they can learn about what it is they are engaging.

The best possibilities of using Wikitude to enhance the learning experience, however, come with their computer software, Wikitude SDK, which allows developers to create their own augmented reality solutions, including image recognition, $2 \mathrm{D}$ and $3 \mathrm{D}$ model rendering, video overlay, location based AR, and several other possibilities, using Javascript or Native API coding languages ("Wikitude SDK"). This means that users and developers who have experience coding can construct their own augmented reality simulations and apply those simulations to real-world structures in order to supplement them with various experiences and, in a way, alter their intended meaning to something entirely different. For example, Gyre at NCMA could become the subject of augmentation from any number of users interested in digital design, literacy, communication and rhetoric, or other fields in the humanities, looking to bridge the gap between the sciences and social sciences. Gyre could be made subject of several enhancements, some of which are already in place at different national parks and sculptures, such as being a place for interactive games, changing the elements around the sculpture, providing narratives (such as the history) or photo galleries, or embedding an online forum with which viewers can engage.

Through the inclusion of augmented reality and some of the capabilities it possesses (as mentioned above), the possibility of material rhetoric discourse could expand upon the inside/outside model to include the proposed "between" concept. In theory, if one were to include "between" in tandem with "inside/outside", what could be explored in material rhetoric could be extended from nature and public spaces to discourse that functions within cyberspace. Augmented reality through the possibilities of Wikitude SDK, then, could offer Gyre an opportunity to become an interactive learning experience connected through naked networks (WiFi, 3g, etc.) which could, in turn, be used by scholars to explore the interaction amongst people/technology/public space/nature, areas often explored by sociologists or critical culture 
theorists/posthumanists, but could also be taken up by those interested in material rhetoric discourse (rhetoricians); emerging technologies such as augmented reality, then, could be used to further explore interdisciplinary studies.

Some applications that exhibit augmented reality are already taking its capabilities to the next level of community engagement, but scholars have yet to consider how they operate via the inside/outside model, let alone with the inclusion of between. Released in July 2016, Niantic's location-based, augmented reality mobile game Pokémon Go utilizes a GPS IP developed in relation with Google Maps to track player's movement throughout the game's digital environment and the player's physical environment. Players are required to travel throughout the real world to progress through the game. In order to catch the virtual creatures found in the game one must physically move themselves, which is tracked via the mobile device's GPS that is tied to the game; as players physically move through the physical/digital environments, they begin to level up/progress by catching more Pokémon, hatching eggs (that will become Pokémon), battling at local gyms, and visiting "Pokéstops" (random stops that players visit for in-game items which are typically related to an important landmark within a community).

As a groundbreaking achievement for video games, due to blending the real world with virtual environments, Pokémon Go offers a prime example of what could be studied rhetorically via inside/outside/between. Because the game's IP is set to geospatially include landmarks around the location of the player (which are utilized as Pokéstops or gyms) whom they can engage, the possibility of examining material rhetoric discourse supplemented with augmented reality offers scholars a new opportunity to examine how physical environments can cross through materiality, nature, and ultimately into networked environments; in those environments, one is enticed to explore how a public space can lead to discourse located in cyberspace. For example, located within the Pokémon Go geocache are several landmarks that have already been explored through material rhetoric discourse, including Gyre, Crossroads/Trickster, Cloud Chamber, The Crown Fountain, and Cloud Gate. Through Niantic's IP, these public sculptures and their spaces have been augmented to become interactive destinations which can be explored through the game (some are gyms that players battle at to take control of, while others are Pokéstops). As a result, these spaces can influence the subject of conversation 
and interaction within cyberspace via naked networks. For example, at the time of this writing, on the subreddit /r/PokémonGoChi there are 10 posts regarding "Millennium Park" and the Pokémon Go community. Of those 8 posts, two contain information on a "Meet Up" (wherein players organize events to put up and experience the game together), five threads offer advice on what you can catch within Millennium Park (or surrounding areas), and the remaining general query about the status of gyms located within the park. Across these 10 threads there are 144 comments with an average of 14 comments per thread ("Millennium Park"). The Triangle of North Carolina (Raleigh/Durham/Cary) also has a subreddit (/r/PokémonGo_Triangle), with hundreds of threads and thousands of posts, although no thread offers information on the North Carolina Museum of Art. Still yet, the North Carolina Museum of Art did become an area of interest for Go players during a "Meet Up" event on September 18, 2016, when several players met at the park to experience the park (and the game) through augmented reality.

The above scenarios, that is, the supplementation of augmented reality on public spaces leading to networked involvement, present how the augmented reality application Pokémon Go can utilize public spaces and natural parks to lead to digital (and physical) experiences. Being public spaces within nature, Gyre, Crossroads/Trickster, Cloud Chamber, The Crown Fountain, and Cloud Gate each operate through the inside/outside mode; that is, they entice the viewer to flow from constructed spaces to natural spaces. Yet, with the possibilities presented by augmentation, and as part of the game's geocache, they enable the viewer to circulate into a wireless network and work between im/materiality by becoming interactive POIs. This would allow scholars to explore material discourse in tandem with digital rhetoric.

One such possibility that could be explored through Pokémon Go, material discourse, and digital rhetoric is examining how "teams" within the game work together to accomplish in-game achievements, such as taking a gym (typically some significant landmark within the player's community - Cloud Gate, for example). This was, and commonly continues to be, the case amongst various Facebook groups dedicated to organizing events based on experiencing the game. The Facebook group "Team Mystic Charleston WV," for example, organizes players of "Team Mystic" (one of the three teams a player selects in Pokémon Go) to march through Charleston and take over gyms 
from opposing teams/players. This is also the case with "Pokémon Go: Official RDU | Team Valor - Team Rocket," an active Facebook group located in Raleigh, North Carolina that employs community engagement and teamwork to take gyms or find virtual Pokémon. In either case, players are organized online because of the game itself, and once organized they band together to subjugate the virtual "public spaces" that the game augments, which are oftentimes embedded in natural history, human history, or both.

\section{Complications and Future Studies}

Augmented reality applications such as Wikitude and Pokémon Go offer rhetorical scholars a chance to go between the lines of the material and natural and into the realm of the digital, while ultimately braiding the three together. Scholars exploring visual rhetoric should begin to consider how augmentation has the capabilities of influencing discourse within the realms of material visual rhetoric and digital rhetoric. That being said, the accessibility of augmented reality is still an important question to raise, as one is seemingly at the mercy of that which is created through programmers or that which already exists. The possibilities of what can be augmented know no bounds, other than being held back by one's ability to code. In a scenario such as how scholars could employ the use of augmented reality for their own studies, it is advisable to learn coding languages that are utilized by programs like Wikitude SDK which employs Javascript and Native (coding languages). Even then, selling the augmented reality application to the public (so that one could explore its impact for study) is another matter, and one that should be considered as well, though they could simply be distributed for free.

Future scholars seeking to explore the inside/outside/between concept should also consider the possibility of linking "between" to "virtual communities" to examine how public spaces, natural spaces, and augmentation can lead to virtual communities/identity. Introduced by David Silver in 2005, a virtual community is that which "connects residents of a specific place -a town, a city, a municipality-with one another and with local institutions, services, and businesses, all via Internet technologies like Websites, e-mail, mailing lists, and bulletin boards" and one that

privileges "interaction, engagement, and participation" within networked environments (188). As examined previously, the inclusion of augmented reality in public spaces has 
transcended beyond materiality and into the digital realm, helping establish the formation of virtual communities (for example, the groups founded through Pokémon Goon both reddit and Facebook). Because augmentation further blends the (already thin) line that exists between digital and physical, scholars could expand interdisciplinary studies from technology, nature, and public spaces to include community identity in both material and virtual settings.

Another approach using the inside/outside/between concept could be in tandem with the literary theory posthumanism. While the theory itself is broad and offers the capabilities to examine the intersection between technology and society in several ways, one such way it could be explored is through hybridization. As presented by Ron Burnett, hybridization is the

[underlying] process of change and evolution and technologies and humans encounter each other. To think in these terms is to put intelligence and the subjective back into human-technology relations. Rather than modeling technology in the broadest sense as a series of tools for pragmatic use, there is a need to think about how human subjectivity and the ability to self-reflexively examine identity has evolved out of the relationships humans have with machinery, artifice, and their creative engagement with technology (Burnett 197).

In other words, hybridization is more than just the intersection and their intertwining, it is about the evolution of these entities changing as they encounter each other. Keeping hybridization in mind during the practice of inside/outside/between could produce scholarship which examines how public spaces, natural spaces, and digital spaces each evolve once they become entangled. Other posthuman works that could be of use include Judith Halberstam's Posthuman Bodies and Katherine Hayles' How We Became Posthuman, each of which explores the human embodiment in relation to the information age. For example, how does embodiment play a role in augmented reality applications, and in what ways does it impact natural, public, and digital spaces?

Last, scholars should continue to examine the inside/outside/between in relation with other material rhetoric discourse strategies and seek to expand those strategies to explore digital design and rhetoric. One such example could be the utilization of 
Gallagher and Zagacki's previously mentioned regenerative/transformative to explore how one travels from a natural state to a human-constructed space, and then back again to the natural state; this could also be expanded upon, wherein one looks at moving from a physical space to a digital space and then back again to the physical. This is typically the scenario with most augmented reality users, as they must be physically present within the space they are augmenting, and, as a result, they travel between the physical and digital realms.

Throughout this research, it has been proposed that exploring digital networks and public and natural spaces could offer insights into the relationship between im/materiality and immateriality. Emerging applications can offer scholars endless possibilities to explore new media and the humanities, as well as the interdisciplinarity between fields such as public spaces, natural spaces, digital spaces, rhetoric, science, technology, critical cultural theory, and several other areas. Although the scope of this paper was limited in its application of augmented reality on physicality, future studies will seek to apply the inside/outside/between model to material structures and then delve into digital discourse, ultimately entangling material structures, nature, and cyberspace together.

\section{Works cited}

Burnett, Ron. How Images Think. The MIT Press, 2004.

Clary-Lemon, Jennifer. "Museums as Material: Experiential Landscapes and the Canadian Museum for Human Rights.” Enculturation, 2015. Accessed 4 Dec. 2016.

Gallagher, Victoria J., and Kenneth Zagacki. "Rhetoric and Materiality in the Museum Park at the North Carolina Museum of Art." Quarterly Journal of Speech, vol. 95, no. 2, 2013.

Gallagher, Victoria J., et al. "Materiality and Urban Communication: The rhetoric of communicative spaces." Communication Matters: Materialist Approaches to Media, Mobility and Networks, edited by Stephen B. Crofts Wiley and Jeremy Packer, Routledge, 2012: 107-119.

Gilfoyle, T.J. Millennium Park: Creating a Chicago Landmark. University of Chicago Press, 2006. 
Liestøl, Gunnar. "Augmented Reality and Digital Genre Design - Situated Simulations on the iPhone." Mixed and Augmented Reality - Arts, Media, and Humanities, 2009. Accessed 25 Nov. 2016.

"Millennium Park." PokémonGoChi. Reddit. 2016. Accesed 13 November 2016.

Silver, David. "Selling Cyberspace: Constructing and Deconstructing the Rhetoric of Community." The Southern Communication Journal, vol. 70, no. 3, 2005.

Sohan, Vanessa. Material Rhetorics and Writing. 2013. Department of English, Florida International University, Miami, FL. PDF file.

Wagner, Ina, Tone Bratteteig, and Dagny Stuedahl, editors. Exploring Digital Design: Multi-Disciplinary Design Practices. Springer-Verlag, 2010. 